\title{
DD ACE gene polymorphism is associated with increased coronary artery endothelial dysfunction: the PREFACE trial
}

\author{
H J G H Mulder, P P van Geel, M J Schalii, W H van Gilst, A H Zwinderman, \\ A V G Bruschke
}

Heart 2003;89:557-558

$E^{n}$ ndothelial dysfunction is an early event in atherogenesis and is associated with the propensity to cause future cardiovascular events. The amelioration of endothelial dysfunction has been a research target for some years now, and success has been reported with, for example, the 3-hydroxy-3-methylglutaryl coenzyme A (HMG-CoA) reductase inhibitors. Research has also identified the homozygous deletion (DD) subset of the angiotensin converting enzyme (ACE) insertion(I)/deletion(D) polymorphism to be associated with deteriorated endothelial function. The results, however, are conflicting. In a substudy of the PREFACE (pravastatin-related effects following angioplasty on coronary endothelium) trial ${ }^{1}$ we assessed ACE polymorphism, coronary endothelial function, and the influence of the HMG-CoA reductase inhibitor pravastatin.

\section{METHODS}

PREFACE $^{1}$ was a randomised, double blinded, placebo controlled, multicentre study designed to assess the effect of three months of treatment with pravastatin $(40 \mathrm{mg}$, once daily) on the endothelial function of native and dilated coronary arteries in non-smoking, non-hypercholesterolaemic patients.

Coronary endothelium dependent vasomotion at three months was assessed by serial, two minute, intracoronary infusions of acetylcholine with the final intracoronary concentrations estimated to be $10^{-8}, 10^{-7}$, and $10^{-6} \mathrm{M}$. Hereafter endothelium independent vasomotion was assessed by the infusion of glyceryl trinitrate at $40 \mu \mathrm{g}$ per minute for two minutes. Within 15 seconds of each infusion, coronary arteriography was performed with a non-ionic contrast medium and a power injector.

Quantitative coronary analysis was performed at the Heartcore angiographic laboratory (Leiden, The Netherlands) in a blinded fashion. Target segments for analysis were the dilated segment and an angiographically normal segment. Segment responses were calculated as the percentage change in the mean segment diameter after infusion relative to the mean segment diameter at baseline.

The ACE genotyping was performed according to a modified procedure from Rigat and associates. ${ }^{2}$

Vasomotion measurements were compared between genotypes by mixed model analysis of variance (ANOVA) with the ACE genotype and acetylcholine infusion dose as fixed factors and the patient number as the random factor. The factors of the model were expanded with treatment and the interaction factor treatment/genotype for analysis of these factors. We also performed a pooled analyses of DD versus ID/II as the literature demonstrates that the DD polymorphism is most often associated with deleterious effects, while the ID/II genotype subsets show similar results.

\section{RESULTS}

Fifty two patients (47 men, 5 women) were included in this substudy. The groups of patients divided according to ACE

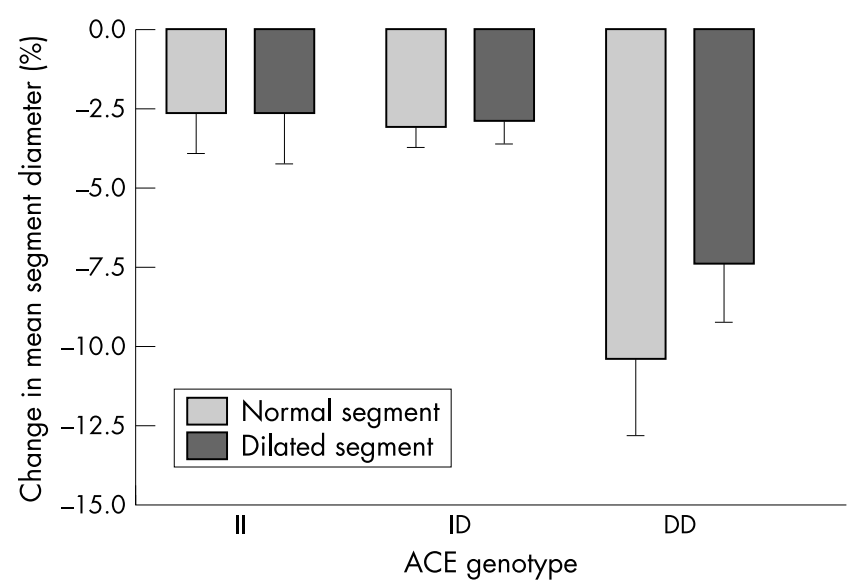

Figure 1 ACE genotype and change in mean segment diameter for normal and dilated coronary segments. DD subset versus the ID/II subsets demonstrates a significant difference in both the dilated $(p=$ $0.003)$ and the normal coronary artery segment groups $(p=0.008)$.

genotype subsets (II, ID, DD) were similar with respect to age, blood pressure, serum lipids, and history of cardiovascular events. The only discernable difference between the groups was the number of patients randomised to pravastatin, which was significantly higher in the ID group—pravastatin/placebo: II (3/7), ID (21/7), DD (6/8).

The luminal parameters of both dilated and normal segments at baseline and after glyceryl trinitrate infusion were similar between the genotype subsets. We found that the DD genotype was associated with a significant deteriorated endothelial function as compared to the other genotypes in both the dilated $(\mathrm{p}=0.03)$ and the normal segment groups $(p=0.01)$. Pooled analyses of DD subset versus the ID/II subset demonstrated increased significance in both the dilated $(p=0.003)$ and the normal coronary artery segment groups $(p=0.008)$ (fig 1$)$. When pravastatin treatment was included in the analyses in the dilated segment group a significant effect was present $(p=0.04)$. When the data are pooled as II/ID versus DD an increased significance of the pravastatin effect is observed $(p=0.02)$.

\section{DISCUSSION}

Perticone and colleagues used forearm strain gauge plethysmography to assess endothelial function in patients with never-treated hypertension. ${ }^{3}$ They observed an association between the DD genotype and endothelial dysfunction. In the normotensive control group, however, no association was
Abbreviations: $A C E$, angiotensin converting enzyme; HMG-CoA, 3-hydroxy-3-methylglutaryl coenzyme A; PREFACE, pravastatin-related effects following angioplasty on coronary endothelium 
present. This led them to the conclusion that the ACE polymorphism did not provide the most important component of endothelial dysfunction. Contrary to these results were the findings of Butler and colleagues ${ }^{4}$ who demonstrated an association between the insertion/deletion and endothelial function in healthy young men.

To our knowledge there are no previous reports on the relation between ACE polymorphism and in vivo epicardial coronary endothelial function. Our results agree with the forearm findings of Butler and colleagues. ${ }^{4}$ We found an association between the DD genotype and deteriorated endothelial function in both normal and dilated coronary artery segments in patients with few risk factors. The dominant influence of the DD genotype is demonstrated by the fact that it is also present in the dilated segments. The endothelium of these segments has been exposed for only two months to factors like hypertension, which exerts its effect in the long term. To our knowledge there are also no previous reports on the relation between ACE genotype, the use of statins, and coronary endothelial function. We found a significant beneficial effect of pravastatin in the DD subset of the dilated segment group.

In summary, this study demonstrates that the DD genotype is associated with blunted endothelial function of epicardial coronary arteries. Furthermore an ameliorating effect of pravastatin in patients with the DD genotype in the dilated coronary artery was found. Although the PREFACE trial was not specifically designed to address the issue of ACE polymorphism effects on endothelial function, we think the results may be of clinical importance and warrants further research.

\section{ACKNOWLEDGEMENTS}

The PREFACE trial was supported by Bristol-Myers Squibb Co, Princeton, New Jersey, USA.

\section{Authors' affiliations}

H J G H Mulder, M J Schalii, A V G Bruschke, Department of Cardiology, Leiden University Medical Center, Leiden, The Netherlands P P van Geel, W H van Gilst, Department of Clinical Pharmacology, University Hospital Groningen, Groningen, The Netherlands A H Zwinderman, Department of Medical Statistics, Leiden University, Leiden, The Netherlands

Correspondence to: Dr Han JGH Mulder, Department of Cardiology, Leiden University Medical Center, Albinusdreef 2, PO Box 9600, 2300 RC, Leiden, The Netherlands; m.j.schalij@lumc.nl

Accepted 20 November 2002

\section{REFERENCES}

1 Mulder HJGH, Schalii M, Kaver B, et al. Pravastatin and endothelium dependent vasomotion after percutaneous transluminal coronary angioplasty (PTCA): the PREFACE (pravastatin related effects following angioplasty on coronary endothelium) trial. Heart 2001;86:533-9.

2 Schunkert H, Hense HW, Holmer SR, et al. Association between a deletion polymorphism of the angiotensin-converting-enzyme gene and left ventricular hypertrophy. N Engl J Med 1994;330:1634-8.

3 Perticone F, Ceravolo R, Maio R, et al. Angiotensin-converting enzyme gene polymorphism is associated with endothelium-dependent vasodilation in never treated hypertensive patients. Hypertension 1998:31:900-5.

4 Butler $\mathbf{R}$, Morris AD, Burchell B, et al. DD angiotensin-converting enzyme gene polymorphism is associated with endothelial dysfunction in normal humans. Hypertension 1999;33:1164-8.

\section{IMAGES IN CARDIOLOGY}

\section{Prolonged sinus arrest after coronary artery spasm}

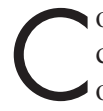

oronary artery spasm has been proposed as a definite cause of vasospastic angina and as a contributor to other acute cardiac events, including unstable angina, acute myocardial infarction, and sudden cardiac death. It has also been demonstrated that silent ischaemia caused by coronary artery spasm could initiate potentially fatal arrhythmias in patients without flow limiting structural coronary artery lesions. We report a case of prolonged sinus arrest after an attack of coronary artery spasm.

A 50 year old woman presented with recurrent spontaneous chest pain that usually occurred in the morning. She had been diagnosed as having hypertension two months previously without taking any medication. Resting ECG showed normal sinus rhythm without ST-T wave change (panel A). Treadmill exercise test with Bruce protocol showed no significant ST change with good exercise tolerance. Holter monitoring was applied, during which the patient had severe chest pain followed by syncope at midnight. Holter monitoring during chest pain showed pronounced ST segment elevation (panel B) followed by bradycardia (panel C). After normalisation of ST segment elevation, prolonged (28 seconds) sinus arrest followed (panel D), which was the cause of her syncope. Coronary angiography showed no significant luminal narrowing. The patient was treated with a calcium channel blocker and nitrates, with a subsequent uneventful clinical course.

J-W Ha S-Y Cho ¡wha@yumc.yonsei.ac.kr
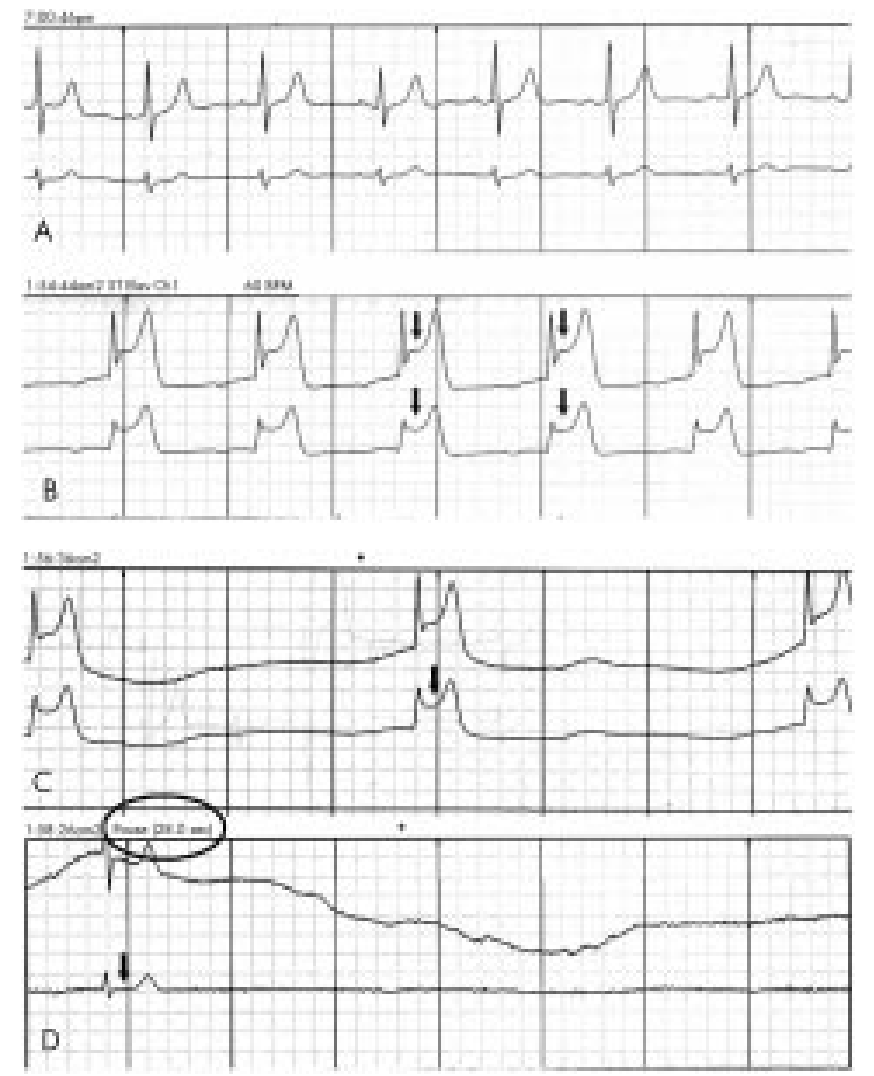